PROCEEDINGS OF THE

AMERICAN MATHEMATICAL SOCIETY

Volume 139, Number 6, June 2011, Pages 1909-1914

S 0002-9939(2011)10920-1

Article electronically published on February 1, 2011

\title{
BUCHSBAUM VARIETIES WITH NEXT TO SHARP BOUNDS ON CASTELNUOVO-MUMFORD REGULARITY
}

\author{
CHIKASHI MIYAZAKI
}

(Communicated by Bernd Ulrich)

\begin{abstract}
This paper is devoted to the study of the next extremal case for a Castelnuovo-type bound $\operatorname{reg} V \leq\lceil(\operatorname{deg} V-1) / \operatorname{codim} V\rceil+1$ of the CastelnuovoMumford regularity for a nondegenerate Buchsbaum variety $V$. A Buchsbaum variety with the maximal regularity is known to be a divisor on a variety of minimal degree if the degree of the variety is large enough. We show that a Buchsbaum variety satisfying $\operatorname{reg} V=\lceil(\operatorname{deg} V-1) / \operatorname{codim} V\rceil$ is a divisor on a Del Pezzo variety if $\operatorname{deg} V \gg 0$.
\end{abstract}

\section{INTRODUCTION}

The Castelnuovo-Mumford regularity is one of the most important invariants measuring a complexity of the defining equations of a projective variety. There have been various studies on bounding the regularity of a variety.

Let $k$ be an algebraically closed field. Let $S=k\left[X_{0}, \cdots, X_{N}\right]$ be the polynomial ring over $k$. A variety $V \subset \mathbb{P}_{k}^{N}=\operatorname{Proj} S$ means a nondegenerate irreducible reduced projective scheme over $k$. For a coherent sheaf $\mathcal{F}$ on $\mathbb{P}_{k}^{N}$ and an integer $m \in \mathbb{Z}$, $\mathcal{F}$ is said to be $m$-regular if $\mathrm{H}^{i}\left(\mathbb{P}_{k}^{N}, \mathcal{F}(m-i)\right)=0$ for all $i \geq 1$. For a projective scheme $X \subseteq \mathbb{P}_{k}^{N}, X$ is said to be $m$-regular if the ideal sheaf $\mathcal{I}_{X}$ is $m$-regular. The Castelnuovo-Mumford regularity of $X \subseteq \mathbb{P}_{k}^{N}$ is the least such integer $m$ and is denoted by reg $X$. A projective scheme $X$ is $m$-regular if and only if for every $p \geq 0$ the minimal generators of the $p$ th syzygy module of the defining ideal $I(=$ $\left.\Gamma_{*} \mathcal{I}_{X} \subset S\right)$ of $X$ occur in degree $\leq m+p ;$ see [3, 4.

For a rational number $m \in \mathbb{Q}$, we write $\lceil m\rceil$ for the minimal integer which is greater than or equal to $m$ and $\lfloor m\rfloor$ for the maximal integer which is less than or equal to $m$.

The Eisenbud-Goto conjecture $\operatorname{reg} V \leq \operatorname{deg} V-\operatorname{codim} V+1$ for a nondegenerate projective variety $V$ is one of the most important problems in this field, and it is widely open to get the bound and the classification of boundary examples in the case of higher dimensional projective varieties. However, if $V$ is an ACM variety, that

Received by the editors May 18, 2009 and, in revised form, March 12, 2010 and March 25, 2010 .

2010 Mathematics Subject Classification. Primary 13H10, 14M05; Secondary 14N25.

Key words and phrases. Castelnuovo-Mumford regularity, Buchsbaum ring, Rational normal scroll, Del Pezzo variety.

The author was partially supported by Grant-in-Aid for Scientific Research (C) (21540044) Japan Society for the Promotion of Science.

(C)2011 American Mathematical Society Reverts to public domain 28 years from publication 
is, the coordinate ring of $V$ is Cohen-Macaulay, then a regularity bound $\operatorname{reg} V \leq$ $\lceil(\operatorname{deg} V-1) / \operatorname{codim} V\rceil+1$ easily follows from the uniform position principle for a generic hyperplane section of a projective curve. Arising from this viewpoint, one has extended the bounding the regularity with invariants coming from the deficiency module of the variety. If $V \subset \mathbb{P}_{k}^{N}$ is a nondegenerate projective curve, then reg $V \leq$ $\lceil(\operatorname{deg} V-1) / \operatorname{codim} V\rceil+\max \{k(V), 1\}$, where $k(V)=\min \left\{v \geq 0 \mid \mathfrak{m}^{v} \mathrm{M}(V)=0\right\}$ and $\mathrm{M}(V)=\mathrm{H}_{*}^{1} \mathcal{I}_{V}$. Further, the boundary curve and the next boundary curve are classified for $\operatorname{deg} V \gg 0$; see [10, 12.

This paper considers a Buchsbaum variety, which is an extended class of an ACM variety. A projective variety $V \subset \mathbb{P}_{k}^{N}$ is called a Buchsbaum variety if the coordinate of $V$ is a Buchsbaum ring. A result of Stückrad and Vogel [18] states that $\operatorname{reg} V \leq\lceil(\operatorname{deg} V-1) / \operatorname{codim} V\rceil+1$ for a nondegenerate Buchsbaum variety $V \subset \mathbb{P}_{k}^{N}$. The extremal case is known to be a divisor on a variety of minimal degree if $\operatorname{deg} V \gg 0$ by Yanagawa for curves [19] and by Nagel [15] in general. In this paper, we classify the next extremal case of the Castelnuovo-type bound of the Castelnuovo-Mumford regularity for a Buchsbaum variety. We show that a Buchsbaum variety $V \subset \mathbb{P}_{k}^{N}$ satisfying $\operatorname{reg} V=\lceil(\operatorname{deg} V-1) / \operatorname{codim} V\rceil$ with $\operatorname{deg} V \gg 0$ is a divisor on a Del Pezzo variety; see Theorem 2.7. More interestingly, our result reminds us of an analogy for the next extremal case of the relationship between the secant lines and the Eisenbud-Goto bound; see [8, §0].

We are grateful to the referee for several stimulating remarks which improved a result of the paper. We would also like to thank Professor Euisung Park for his helpful comments.

\section{Castelnuovo-Mumford Regularity of Buchsbaum varieties}

First we recall the definition of a Buchsbaum variety. For background information concerning Buchsbaum rings we refer to the book [17.

Let $S=k\left[x_{0}, \cdots, x_{N}\right]$ be the polynomial ring over $k$ with the homogeneous maximal ideal $\mathfrak{m}$. Let $V\left(\subset \mathbb{P}_{k}^{N}=\operatorname{Proj} S\right)$ be a projective scheme. Let $I=\Gamma_{*} \mathcal{I}_{V / \mathbb{P}^{N}}$ be the defining ideal of $V$. Let $R=S / I$ be the coordinate ring of $V$ and put $\mathfrak{n}=\mathfrak{m} / I$. The deficiency module of $V$ is defined as $\mathrm{M}^{i}(V)=\mathrm{H}_{*}^{i} \mathcal{I}_{V / \mathbb{P}^{N}}=\bigoplus_{\ell \in \mathbb{Z}} \mathrm{H}^{i}\left(\mathcal{I}_{V / \mathbb{P}^{N}}(\ell)\right)$ for $i=1, \cdots, \operatorname{dim} V$; see, e.g., [9].

Definition 2.1. The projective scheme $V$ is called a quasi-Buchsbaum scheme if $\mathfrak{m M}^{i}(V)=0$ holds for $i=1, \cdots, \operatorname{dim} V$. The projective scheme $V$ is called a Buchsbaum scheme if $R_{\mathfrak{n}}$ is a Buchsbaum ring. In case $V$ is irreducible and reduced, we call $V$ a Buchsbaum variety.

Proposition 2.2. Let $V\left(\subset \mathbb{P}_{k}^{N}=\operatorname{Proj} S\right)$ be a projective scheme with $\operatorname{dim} V=$ $n$. The scheme $V$ is a Buchsbaum scheme if and only if for any hyperplane $H_{1}, \cdots, H_{n-1}$ satisfying that $\operatorname{dim} V_{j}=\operatorname{dim} V-j$, where $V_{n-j}=V \cap H_{1} \cap \cdots \cap H_{j}$ for $j=0, \cdots, n-1$, the scheme $V_{j}$ is quasi-Buchsbaum.

Remark 2.3. We simply call it a Buchsbaum variety in this paper, while it is called an arithmetically Buchsbaum variety in [15, 19. If $V$ is a Buchsbaum variety, then a generic hyperplane section of $V$ is also a Buchsbaum variety.

In this section we investigate a Castelnuovo-type bound for the CastelnuovoMumford regularity for Buchsbaum varieties. Let us start by stating a result of Stückrad-Vogel [18]. 
Proposition 2.4. Let $V$ be a Buchsbaum variety of $\mathbb{P}_{k}^{N}$ with $n=\operatorname{dim} V \geq 1$ over an algebraically closed field. Let $W=V \cap H$ be a generic hyperplane section. Then we have $\operatorname{reg} W=\operatorname{reg} V$. Consequently, $\operatorname{reg} V \leq\lceil(\operatorname{deg} V-1) / \operatorname{codim} V\rceil+1$.

In general, a nondegenerate projective variety $V \subset \mathbb{P}_{k}^{N}$ satisfies $\operatorname{deg} V \geq \operatorname{codim} V$ +1 . The projective variety $V$ is called a variety of minimal degree if $\operatorname{deg} V=$ $\operatorname{codim} V+1$. In this case, the variety $V$ is classified to be a hyperquadric, a (cone over the) Veronese surface, or a rational normal scroll; see [6, (3.10)] and [5, (5.10)]. A nondegenerate projective variety $V$ is a variety of almost minimal degree if $\operatorname{deg} V=\operatorname{codim} V+2$, which is classified to be either a normal Del Pezzo variety or the image of a variety of minimal degree via a projection; see [2, 5, 16.

The following result describes Buchsbaum varieties with the maximal regularity of Castelnuovo-type.

Proposition 2.5 ([15, 19]). Let $V \subseteq \mathbb{P}_{k}^{N}$ be a nondegenerate Buchsbaum variety over an algebraically closed field $k$ with $\operatorname{char} k=0$ or $\operatorname{codim} V \geq 5$. If $\operatorname{deg} V \geq$ $(\operatorname{codim} V)^{2}+2 \operatorname{codim} V+2$ and $\operatorname{reg} V=\lceil(\operatorname{deg} V-1) / \operatorname{codim} V\rceil+1$, then $V$ is a divisor on a variety of minimal degree.

The converse of (2.5) is also obtained by Nagel [15, (4.2)].

Lemma 2.6. If a nondegenerate Buchsbaum variety $V \subseteq \mathbb{P}_{k}^{N}$ is a divisor on a variety of minimal degree, then $\operatorname{reg} V=\lceil(\operatorname{deg} V-1) / \operatorname{codim} V\rceil+1$.

Let $S=k\left[X_{0}, \cdots, X_{N}\right]$ be the polynomial ring over a field $k$ with the homogeneous maximal ideal $\mathfrak{m}=S_{+}$. Let $M$ be a finitely generated graded $S$-module. Then we define $a_{-}(M)=\min \left\{\ell \mid[M]_{\ell} \neq 0\right\}$. Also, we define the socle of $M$ as $\operatorname{Soc}(M)=\{x \in M \mid \mathfrak{m} x=0\}$, which is a graded $S$-module; see, e.g., [7.

We will study Buchsbaum varieties with next to sharp bounds of Castelnuovotype on the Castelnuovo-Mumford regularity.

Theorem 2.7. Let $V \subset \mathbb{P}_{k}^{N}$ be a nondegenerate Buchsbaum variety over an algebraically closed field $k$ with char $k=0$. Assume $\operatorname{deg} V \geq(\operatorname{codim} V)^{2}+4 \operatorname{codim} V+2$. If $\operatorname{reg} V=\lceil(\operatorname{deg} V-1) / \operatorname{codim} V\rceil$, then $V$ is a divisor on a Del Pezzo variety.

Proof. Let $n=\operatorname{dim} V$. Let us take generic hyperplanes $H_{1}, \cdots, H_{n}$. Let us define $V_{n-j}=V \cap H_{1} \cap \cdots \cap H_{j}$ and $L_{n-j}=H_{1} \cap \cdots \cap H_{j}$ for $j=0, \cdots, n$. Then $\operatorname{dim} V_{i}=i$ and $L_{i} \cong \mathbb{P}_{k}^{N-n+i}$ for $i=0, \cdots, n$. From (2.4), we have $\operatorname{reg} V_{0}=\operatorname{reg} V_{1}=\cdots=$ $\operatorname{reg} V_{n}$. So, reg $V_{0}=\left\lceil\left(\operatorname{deg} V_{0}-1\right) / \operatorname{codim} V_{0}\right\rceil$. By [12, (2.3)] and Lemma 2.6, $V_{0}$ lies on an elliptic normal curve $Y_{0}$. By an elliptic normal curve we mean an irreducible nondegenerate ACM curve of arithmetic genus one. The defining equations of an elliptic normal curve consist of quadric equations except for the case $Y_{0}$ of a plane cubic curve.

Let $c=\operatorname{codim} V=\operatorname{codim} V_{0}$ and $d=\operatorname{deg} V=\operatorname{deg} V_{0}$. Then we see $\operatorname{deg} Y_{0}=$ codim $Y_{0}+2=c+1$.

Now we will show that if $c \geq 3$, then $\Gamma\left(\mathcal{I}_{Y_{0}}(2)\right) \cong \Gamma\left(\mathcal{I}_{V_{0}}(2)\right)$. Indeed, if there exists a hyperquadric $Q$ such that $V_{0} \subseteq Q$ and $Y_{0} \nsubseteq Q$, then $V_{0} \subseteq Y_{0} \cap Q$ and $d \leq 2(c+1)$ by the Bezout theorem, which contradicts the assumption $d \geq c^{2}+4 c+2$. On the other hand, if $c=2$, that is, $Y_{0}$ is a plane cubic curve, then $\Gamma\left(\mathcal{I}_{Y_{0}}(3)\right) \cong$ $\Gamma\left(\mathcal{I}_{V_{0}}(3)\right)$. Indeed, if not, we similarly obtain an inequality $d \leq 3(c+1)=9$, which contradicts the assumption $d \geq 14$. 
In order to prove that $\Gamma\left(\mathcal{I}_{V_{1}}(2)\right) \rightarrow \Gamma\left(\mathcal{I}_{V_{0}}(2)\right)$ is surjective for $c \geq 3$ and that $\Gamma\left(\mathcal{I}_{V_{1}}(3)\right) \rightarrow \Gamma\left(\mathcal{I}_{V_{0}}(3)\right)$ is surjective for $c=2$, we have only to show $\mathrm{H}^{1}\left(\mathcal{I}_{V_{1}}(1)\right)=0$ and $\mathrm{H}^{1}\left(\mathcal{I}_{V_{1}}(2)\right)=0$ respectively.

The exact sequence $\mathrm{H}_{*}^{1}\left(\mathcal{I}_{V_{1}}(-1)\right) \stackrel{h}{\rightarrow} \mathrm{H}_{*}^{1}\left(\mathcal{I}_{V_{1}}\right) \rightarrow \mathrm{H}_{*}^{1}\left(\mathcal{I}_{V_{0}}\right)$ leads to an injective map $\mathrm{H}_{*}^{1}\left(\mathcal{I}_{V_{1}}\right) \rightarrow \operatorname{Soc}\left(\mathrm{H}_{*}^{1}\left(\mathcal{I}_{V_{0}}\right)\right)$ because $V_{1}$ is a Buchsbaum variety and $\mathfrak{m H}_{*}^{1}\left(\mathcal{I}_{V_{1}}\right)=0$. So, let us study the structure of $\operatorname{Soc}\left(\mathrm{H}_{*}^{1}\left(\mathcal{I}_{V_{0}}\right)\right)$ in the positive graded part as an $S$-graded module. Since $Y_{0}$ is ACM, we have the exact sequence $\mathrm{H}_{*}^{1}\left(\mathcal{I}_{Y_{0}}\right)=0 \rightarrow \mathrm{H}_{*}^{1}\left(\mathcal{I}_{V_{0}}\right) \rightarrow$ $\mathrm{H}_{*}^{1}\left(\mathcal{I}_{V_{0} / Y_{0}}\right) \rightarrow \mathrm{H}_{*}^{2}\left(\mathcal{I}_{Y_{0}}\right)$. Note that $\mathrm{H}^{2}\left(\mathcal{I}_{Y_{0}}(\ell)\right) \cong \mathrm{H}^{1}\left(\mathcal{O}_{Y_{0}}(\ell)\right) \cong\left(\mathrm{H}^{0}\left(\mathcal{O}_{Y_{0}}(-\ell)\right)\right)^{\prime}=0$ for $\ell>0$. Thus we have $\operatorname{Soc}\left(\mathrm{H}_{*}^{1}\left(\mathcal{I}_{V_{0}}\right)\right)=\operatorname{Soc}\left(\mathrm{H}_{*}^{1}\left(\mathcal{I}_{V_{0} / Y_{0}}\right)\right)$ in the positive graded part.

Note that $V_{0}$ may contain a singular point of $Y_{0}$, which happens only in the case where $Y_{0}$ is a rational curve with $p_{a}\left(Y_{0}\right)=1$. If not, we see that $\mathcal{I}_{V_{0} / Y_{0}} \cong \mathcal{O}_{Y_{0}}\left(-V_{0}\right)$. In this case, by Serre duality, the graded $S$-module $\operatorname{Soc}\left(\mathrm{H}_{*}^{1}\left(\mathcal{I}_{V_{0} / Y_{0}}\right)\right)$ is isomorphic to the dual of $\Gamma_{*}\left(\mathcal{O}_{Y_{0}}\left(V_{0}\right)\right) / \mathfrak{m} \Gamma_{*}\left(\mathcal{O}_{Y_{0}}\left(V_{0}\right)\right)$. Let $\mathcal{F}=\mathcal{O}_{Y_{0}}\left(V_{0}\right)$. Then we have $\mathrm{H}^{1}\left(\mathcal{F} \otimes \mathcal{O}_{Y_{0}}(m-1)\right)=0$ for $d+(c+1)(m-1) \geq 1$. In other words, $\mathcal{F}$ is $m$ regular for $m \geq(c-d+2) /(c+1)$. Let us put $m=\lceil(c-d+2) /(c+1)\rceil$. Then $\Gamma\left(\mathcal{F} \otimes \mathcal{O}_{Y_{0}}(\ell)\right) \otimes \Gamma\left(\mathcal{O}_{Y_{0}}(1)\right) \rightarrow \Gamma(\mathcal{F}(\ell+1))$ is surjective for $\ell \geq m$ by [13. Hence we obtain $a_{-}\left(\operatorname{Soc}\left(\mathrm{H}_{*}^{1} \mathcal{I}_{V_{0} / Y_{0}}\right)\right) \geq-m$.

Next assume that $Y_{0}$ is singular and $V_{0}$ contains a singular point of $Y_{0}$. The normalization $\pi: \tilde{Y}_{0} \rightarrow Y_{0}$ is indeed a one-point linear projection of a rational normal curve $\tilde{Y}_{0}$. Let $\tilde{V}_{0}=\pi^{*}\left(V_{0}\right)$. Then we have $\mathrm{H}_{*}^{1}\left(\mathcal{I}_{V_{0} / Y_{0}}\right) \cong \mathrm{H}_{*}^{1}\left(f_{*} f^{*} \mathcal{I}_{V_{0} / Y_{0}}\right) \cong$ $\mathrm{H}_{*}^{1}\left(\mathcal{I}_{\tilde{V}_{0} / \tilde{Y}_{0}}\right)$. Let $\mathcal{F}=\mathcal{O}_{\tilde{Y}_{0}}\left(\tilde{V}_{0}\right)$. Note that $\tilde{Y}_{0}$ is a rational normal curve. Thus we have $\mathrm{H}^{1}\left(\mathcal{F} \otimes \mathcal{O}_{\tilde{Y}_{0}}(m-1)\right)=0$ for $d+1+(c+1)(m-1) \geq-1$. From the previous argument, we have $a_{-}\left(\operatorname{Soc}\left(\mathrm{H}_{*}^{1} \mathcal{I}_{V_{0} / Y_{0}}\right)\right) \geq-m$, where $m=\lceil(c-d-1) /(c+1)\rceil$.

Conclusively, we see that $a_{-}\left(\operatorname{Soc}\left(\mathrm{H}_{*}^{1}\left(\mathcal{I}_{V_{0}}\right)\right)\right) \geq 2$ if $d \geq 3 c+4$ and that $a_{-}\left(\operatorname{Soc}\left(H_{*}^{1}\left(\mathcal{I}_{V_{0}}\right)\right)\right) \geq 3$ if $d \geq 4 c+5$. Since $d \geq c^{2}+4 c+2$, we obtain $\mathrm{H}^{1}\left(\mathcal{I}_{V_{1}}(1)\right)=0$ for $c \geq 3$ and $\mathrm{H}^{1}\left(\mathcal{I}_{V_{1}}(2)\right)=0$ for $c=2$.

For $c \geq 3$, we have a surjective map $\Gamma\left(\mathcal{I}_{V_{1}}(2)\right) \rightarrow \Gamma\left(\mathcal{I}_{V_{0}}(2)\right) \cong \Gamma\left(\mathcal{I}_{Y_{0}}(2)\right)$. Note that $Y_{0}$ is the intersection of the hyperquadrics of $L_{0}\left(\cong \mathbb{P}_{k}^{N-n}\right)$ containing $V_{0}$. Let $Y_{1}^{\prime}$ be the intersection of the hyperquadrics of $L_{1}\left(\cong \mathbb{P}_{k}^{N-n+1}\right)$ containing $V_{1}$. Since $Y_{1}^{\prime} \cap H_{1}=Y_{0}$, there is an irreducible component $Y_{1}$ of $Y_{1}^{\prime}$ such that $Y_{1} \cap H_{1}=Y_{0}$ and $Y_{1}$ is nondegenerate. For $c=2$, we are similarly done as in the case $c \geq 3$. Hence there exists a surface $Y_{1}$ containing $V_{1}$ such that $Y_{1} \cap H_{0}=Y_{0}$, $\operatorname{codim} Y_{1}=\operatorname{codim} Y_{0}$ and $\operatorname{deg} Y_{1}=\operatorname{deg} Y_{0}$.

Let $1 \leq i \leq n-1$. Assume that $V_{i}$ is a divisor on a variety $Y_{i}$ of almost minimal degree. We will proceed to construct inductively a variety $Y_{i+1}$ of almost minimal degree containing $V_{i+1}$. As in the case of $i=0$, we need to show that $\Gamma\left(\mathcal{I}_{V_{i+1}}(2)\right) \rightarrow \Gamma\left(\mathcal{I}_{V_{i}}(2)\right)$ is surjective for $c \geq 3$. By the inductive process, $\mathrm{H}^{1}\left(\mathcal{I}_{V_{i}}(1)\right)=0$ implies $\mathrm{H}^{1}\left(\mathcal{I}_{V_{i+1}}(1)\right)=0$, which gives the surjectivity of $\Gamma\left(\mathcal{I}_{V_{i+1}}(2)\right)$ $\rightarrow \Gamma\left(\mathcal{I}_{V_{i}}(2)\right)$. For $c=2$, we can similarly show that $\Gamma\left(\mathcal{I}_{V_{i+1}}(3)\right) \rightarrow \Gamma\left(\mathcal{I}_{V_{i}}(3)\right)$ is surjective. By the same method as for $i=0$, we have a nondegenerate projective variety $Y_{i+1} \subset L_{i+1}\left(\cong \mathbb{P}_{k}^{N-n+i+1}\right)$ with $Y_{i+1} \cap H_{i}=Y_{i}$ and $\operatorname{deg} Y_{i+1}=\operatorname{deg} Y_{i}$ such that $V_{i+1}$ is a divisor on $Y_{i+1}$ for $i=1, \cdots, n-1$. The variety $Y_{i}$ can be easily shown to be a Del Pezzo variety by induction on $i$ from [5, (1.6.4.5)]. Hence the assertion is proved.

Remark 2.8. For a nondegenerate Buchsbaum variety $V \subseteq \mathbb{P}_{k}^{N}$ with char $k=0$ which is not a divisor on any variety of minimal degree, if $\operatorname{deg} V \geq 2(\operatorname{codim} V+1)^{2}$, 
then $\operatorname{reg} V<\lceil(\operatorname{deg} V+1) /(\operatorname{codim} V+1)\rceil$ by [15, (4.3)]. As a result, there are no Buchsbaum varieties $V \subseteq \mathbb{P}_{k}^{N}$ with $(\operatorname{codim} V)^{2}+4 \operatorname{codim} V+2 \leq \operatorname{deg} V \leq$ $2(\operatorname{codim} V+1)^{2}-1$ such that $\operatorname{reg} V=\lceil(\operatorname{deg} V+1) /(\operatorname{codim} V+1)\rceil$.

Remark 2.9. The assumption char $k=0$ is necessary for the use of [12, (2.3)] in this proof. However, if $V_{0}$ is in uniform position, then [12, (2.3)] works for char $k>0$; see, e.g. [14, (4.1)]. Although a generic hyperplane section $V_{0}$ of a projective curve may not be in uniform position for char $k>0$, the Castelnuovo's method as in [1, 11] gives reg $V_{0} \leq\left\lceil\left(\operatorname{deg} V_{0}-1\right) / \operatorname{codim} V_{0}\right\rceil-1$ if $\operatorname{deg} V_{0} \gg 0$. Thus the corresponding statement of (2.7) works without describing the degree condition explicitly. "For a nondegenerate Buchsbaum variety $V \subset \mathbb{P}_{k}^{N}$ with $\operatorname{deg} V \gg 0$ and $\operatorname{codim} V \geq 3$, if $\operatorname{reg} V=\lceil(\operatorname{deg} V-1) / \operatorname{codim} V\rceil$, then $V$ is a divisor on a Del Pezzo variety."

Finally we describe examples of nondegenerate non-ACM Buchsbaum curves $V$ with $\operatorname{reg} V=\lceil(\operatorname{deg} V-1) / \operatorname{codim} V\rceil$ on a classical Del Pezzo surface.

Example 2.10 ([12, (4.2), (4.3)]). Let $Y=\mathbb{P}_{k}^{1} \times \mathbb{P}_{k}^{1}$. We write $\mathcal{O}_{Y}(a, b)$ for $\pi_{1}^{*} \mathcal{O}_{Y}(a) \otimes \pi_{2}^{*} \mathcal{O}_{Y}(b)$, where $\pi_{1}$ and $\pi_{2}$ are the first and second projections respectively. Let $Z_{1}$ and $Z_{2}$ be divisors corresponding to $\mathcal{O}_{Y}(1,0)$ and $\mathcal{O}_{Y}(0,1)$ respectively. Through a 2-uple embedding of $Y$ by $H=2 Z_{1}+2 Z_{2}, Y$ is a Del Pezzo surface of degree 8 in $\mathbb{P}_{k}^{8}$. Let $V$ be a divisor on $Y$ linearly equivalent to $a Z_{1}+b Z_{2}$, where $a \leq b$. Then $V$ is a non-ACM Buchsbaum variety with $\operatorname{reg} V=\lceil(\operatorname{deg} V-1) / \operatorname{codim} V\rceil$ if and only if $(a, b)=(2 m, 2 m+2)$ for $5 \leq m \leq 11$, $(a, b)=(2 m, 2 m+3)$ for $3 \leq m \leq 9,(a, b)=(2 m+1,2 m+4)$ for $6 \leq m \leq 12$ or $(a, b)=(2 m+1,2 m+5)$ for $4 \leq m \leq 10$.

Let $\pi: Y=\mathbb{P}(\mathcal{E}) \rightarrow \mathbb{P}_{k}^{1}$ be a projective bundle, where $\mathcal{E}=\mathcal{O}_{\mathbb{P}_{k}^{1}} \oplus \mathcal{O}_{\mathbb{P}_{k}^{1}}(-1)$. Let $Z$ be a minimal section and $F$ be a fiber of $\pi$. Through an embedding of $Y$ by $H=2 Z+3 F, Y$ is a Del Pezzo surface of degree 8 in $\mathbb{P}_{k}^{8}$. Let $V$ be a divisor on $Y$ linearly equivalent to $a Z+b F$. Then $V$ is a non-ACM Buchsbaum variety with $\operatorname{reg} V=\lceil(\operatorname{deg} V-1) / \operatorname{codim} V\rceil$ if and only if $(a, b)=(2 m, 3 m+2)$ for $5 \leq m \leq 11$, $(a, b)=(2 m+1,3 m+3)$ for $2 \leq m \leq 8$ or $(a, b)=(2 m+1,3 m+4)$ for $7 \leq m \leq 13$.

\section{REFERENCES}

[1] E. Ballico and C. Miyazaki, Generic hyperplane section of curves and an application to regularity bounds in positive characteristic, J. Pure Appl. Algebra 155 (2001), 93 - 103. MR.1801407 (2001m:14006)

[2] M. Brodmann and P. Schenzel, Arithmetic properties of projective varieties of almost minimal degree, J. Algebraic Geometry 16 (2007), 347 - 400. MR2274517(2008b:14085)

[3] D. Eisenbud and S. Goto, Linear free resolutions and minimal multiplicity, J. Algebra 88 (1984), 89 - 133. MR741934 (85f:13023)

[4] D. Eisenbud, The geometry of syzygies, GTM 229, Springer, 2005. MR2103875 (2005h:13021)

[5] T. Fujita, Classification theories of polarized varieties, London Math. Soc. Lecture Note Series 155, Cambridge University Press, 1990. MR.1162108 (93e:14009)

[6] J. Harris (with D. Eisenbud), Curves in projective space, Les Presses de l'Université de Montréal, 1982. MR0685427 (84g:14024)

[7] C. Huneke and B. Ulrich, General hyperplane sections of algebraic varieties, J. Algebraic Geometry 2 (1993), 487 - 505. MR 1211996 (94b:14046)

[8] S. Kwak, Smooth projective varieties with extremal or next to extremal curvilinear secant subspaces, Trans. Amer. Math. Soc. 357 (2005), 3553 - 3566. MR2146638 (2006e:14072)

[9] J. C. Migliore, Introduction to liaison theory and deficiency modules, Progress in Math. 165, Birkhäuser, 1998. MR1712469 (2000g:14058)

[10] C. Miyazaki, Sharp bounds on Castelnuovo-Mumford regularity, Trans. Amer. Math. Soc. 352 (2000), 1675 - 1686. MR 1621769 (2000i:13017) 
[11] C. Miyazaki, Castelnuovo-Mumford regularity and classical method of Castelnuovo, Kodai Math. J. 29 (2006), 237 - 247. MR2247433 (2007f:14057)

[12] C. Miyazaki, Projective curves with next to sharp bounds on Castelnuovo-Mumford regularity, J. Algebra 315 (2007), 279 - 285. MR2344346 (2008k:14050)

[13] D. Mumford, Lectures on curves on an algebraic surface, Annals of Math. Studies 59, Princeton Univ. Press, 1966. MR0209285 (35:187)

[14] U. Nagel, On the defining equations and syzygies of arithmetically Cohen-Macaulay varieties in arbitrary characteristic, J. Algebra 175 (1995), 359 - 372. MR.1338983 (96f:13023)

[15] U. Nagel, Arithmetically Buchsbaum divisors on varieties of minimal degree, Trans. Amer. Math. Soc. 351 (1999), 4381-4409. MR1615938(2000a:14058)

[16] E. Park, Smooth varieties of almost minimal degree, J. Algebra 314 (2007), 185 - 208. MR2331758 (2009c:14108)

[17] J. Stückrad and W. Vogel, Buchsbaum rings and its applications, Springer, 1986. MR881220 (88h:13011a)

[18] J. Stückrad and W. Vogel, Castelnuovo bounds for certain subvarieties in $P^{n}$, Math. Ann. 276 (1987), 341 - 352. MR870972 (88e:13013)

[19] K. Yanagawa, On the regularities of arithmetically Buchsbaum curves, Math. Z. 226 (1997), 155 - 163. MR:1472146 (99b:14028)

Department of Mathematics, Saga University, Honjo-machi 1, Saga 840-8502, Japan

E-mail address: miyazaki@ ms.saga-u.ac.jp 